a real-space image of the property under investigation. Obviously, the inversion algorithm and the data analysis in general are key for this method. In the present study, Watari et al. have employed coherent X-ray scattering to characterize AuNPs coated with self-assembled monolayers (see Fig. 1). It turns out that coherent $\mathrm{X}$-ray diffraction is extremely sensitive to displacements of the atoms from their equilibrium structure, and that even the small contribution of chemically induced strain on the nearsurface region can be visualized through difference maps. Such maps are basically the comparison between an unperturbed and a perturbed system and suitable to identify localized small changes.

They find that the structure of a single 300-nm-diameter facetted gold nanocrystal changes profoundly after the adsorption of thiols. The changes require large stresses comparable to those observed in cantilever measurements. Watari et al. conclude that the stress generated by thiol adsorption on gold has a fundamentally different nature in the curved, nominally spherical, regions of the crystal surface than its flat facets. This seems to be a major step, considering that already the interface structure, that is, the binding geometry, of thiols on flat surfaces has been a subject of substantial controversy.

The characterization of the atomic-level structure should bring us one step further in our understanding of nanoparticles. At the same time, it is a beautiful demonstration of the power of coherent X-ray diffraction, which is still seen as a specialist technique and some distance away from being applied routinely. With the increasing availability of coherent sources, in particular the new $\mathrm{X}$-ray lasers, more exciting applications of this technique lie ahead.

Frank Schreiber is at the Institut für Angewandte Physik, Universität Tübingen, Auf der Morgenstelle 10, 72076 Tübingen, Germany.

e-mail: frank.schreiber@uni-tuebingen.de

References

1. Liu, N., Tang, M. L., Hentschel, M., Giessen, H. \& Alivisatos, A. P. Nature Mater. 10, 631-636 (2011)

2. Zhang, D. et al. Phys. Rev. Lett. 104, 056601 (2010).

3. Min, Y., Akbulut, M., Kristiansen, K., Golan, Y. \& Israelachvili, J. Nature Mater. 7, 527-538 (2008).

4. Schreiber, F. Prog. Surf. Sci. 65, 151-256 (2000).

5. Watari, M. et al. Nature Mater. 10, 862-866 (2011).

6. Feidenhans'l, R. Surf. Sci. Rep. 10, 105-188 (1989).

7. Pfeifer, M. A., Williams, G. J., Vartanyants, I. A., Harder, R. \& Robinson, I. K. Nature 442, 63-66 (2006).

\section{A QUICK FIX}

Delicately patterned micro- and nanostructures can be produced without the need for laborious fabrication, by relying on physical forces to organize materials spontaneously into intricate forms. The capacity of soft materials such as surfactants and block copolymers to self-organize into regular patterns has been long recognized ${ }^{1-3}$, and superlattices and other regular arrays in two and three dimensions can be produced from the microphase separation of block copolymers ${ }^{4-6}$. Many of these approaches recognize the precedence of nature, which uses the same parsimonious principles to make elaborate micropatterns such as exoskeletons ${ }^{7}$ and photonic crystals ${ }^{8,9}$.

Not all of the rich patterns generated spontaneously by physical forces are long-lived, however, which may limit opportunities for exploiting them in technologies. Fluids are particularly apt to display transient patterned instabilities that are gone in an instant. Yet an instant is more or less all it takes to 'freeze' such structures in a method described by Grilli et al., who have used rapid heat curing of a liquid polymer to create a range of transparent microstructures that might find uses in optics ${ }^{10}$.

The researchers have focused primarily on the 'beading' of a liquid filament - a variant of the Rayleigh instability studied since the late nineteenth century. This describes the way a column of liquid will become spontaneously unstable to undulatory perturbations that break it up into a string of roughly spherical droplets, often of alternating size. The instability can be seen in a narrow liquid jet, and it is responsible for the way the glue coating the threads of a spider's web forms a string of beads. Dew does likewise, decorating the web with a beautiful array of droplets.

Grilli et al. induce the patterning artificially by using an electrohydrodynamic method ${ }^{11}$ in which a strong electric-field gradient produces instabilities in a dielectric fluid, here the molten polymer polydimethylsiloxane. In a typical example, a thin liquid bridge is formed by using the field between two plates to draw up a droplet of polymer sitting on the lower plate. The bridge drains to a very narrow column that then bunches into 'beads on a string.' A hot-air jet cures the structure within seconds, before it can fragment. The result is a string of linked, transparent, spherical or lenticular beads just a few or a few tens of micrometres across. These can be used as, for example, one-dimensional arrays of optical resonators, with optical properties tunable by mixing fluorescent particles into the liquid polymer.

Other 'frozen' instabilities that may be created this way include 'axicons': conical structures with a needle tip at their apex, which might be used as elements for optical tweezers with a

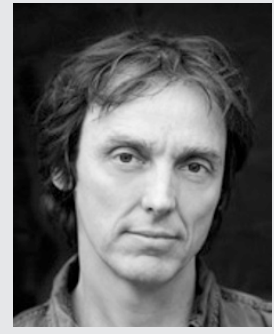

PHILIP BALL

large depth of focus, or as the tips of near-field optical microscopes.

The idea of using the self-organizing forces in liquids to create devices too small to be easily fabricated by hand is actually a very old one. It is how Antoni van Leeuwenhoek made the tiny lenses for the microscopes that enabled him to launch the field of microbiology, by rapidly cooling molten glass into tiny spherical beads.

\section{References}

1. Hyde, S. et al. The Language of Shape (Elsevier, 1996).

2. Fredrickson, G. H. \& Bates, F. S. Annu. Rev. Mater. Sci. 26, 501-550 (1996).

3. Kresge, C. T., Leonowicz, M. E., Roth, W. J., Vartuli, J. C. \& Beck, J. S. Nature 359, 710-712 (1992).

4. Cheng, J. Y., Mayes, A. M. \& Ross, C. A. Nature Mater 3, 823-828 (2004).

5. Park, S. et al. Science 323, 1030-1033 (2009).

6. Lin, Y. et al. Nature 434, 55-59 (2005).

7. Mann, S. \& Ozin, G. A. Nature 382, 313-318 (1996)

8. Michielsen, K. \& Stavenga, D. G. J. R. Soc. Interface 5, 85-94 (2008).

9. Vukusic, P. \& Sambles, J. R. Nature 424, 852-855 (2003).

10. Grilli, S. et al. Proc. Natl Acad. Sci. USA 108, 15106-15111 (2011).

11. Schäffer, E., Thurn-Albrecht, T., Russell, T. P. \& Steiner, U. Nature 403, 874-877 (2000) 